\title{
Missing conformers. Comparative study of conformational cooling in cyanoacetic acid and methyl cyanoacetate isolated in low temperature inert gas matrixes
}

\author{
I.D. Reva $^{\text {a,* }}$, S.G. Stepanian ${ }^{\text {b }}$, L. Adamowicz ${ }^{\text {c }}$, R. Fausto ${ }^{\text {a }}$ \\ a Department of Chemistry, University of Coimbra, Rua Larga, Coimbra 3004-535, Portugal \\ ${ }^{\mathrm{b}}$ Institute for Low Temperature Physics and Engineering, National Academy of Sciences of Ukraine, \\ 47 Lenin Ave., Kharkov 61164, Ukraine \\ ${ }^{\mathrm{c}}$ Department of Chemistry, University of Arizona, Tucson, AZ 85721, USA
}

Received 13 March 2003; in final form 6 May 2003

\begin{abstract}
A comparative conformational study of two related systems, methyl cyanoacetate (MCA) and cyanoacetic acid (CAA), is presented. Ab initio calculations predicted that both systems have two nearly isoenergetic conformers separated by similar low energy barriers (about $3 \mathrm{~kJ} \mathrm{~mol}^{-1}$ ). In xenon matrixes deposited at temperatures above $40 \mathrm{~K}$ for MCA and above $20 \mathrm{~K}$ for CAA only one conformer was observed for each of the two systems. However, below those temperatures two MCA and two CAA conformers were trapped into the matrixes. Conformational cooling was found responsible for this behavior. Factors contributing to this effect are discussed.
\end{abstract}

(c) 2003 Elsevier Science B.V. All rights reserved.

\section{Introduction}

The low temperature matrix isolation is a powerful tool in conformational studies of nonrigid molecules. A low temperature matrix is a non-equilibrium system, where internal rotations in the studied molecules are, in general, hindered due to the interaction with the solid environment. This makes trapping of higher energy species in the matrix and their subsequent identification by spectroscopic methods possible. Most frequently, the distribution of conformers in the matrix cor-

\footnotetext{
${ }^{*}$ Corresponding author. Fax: +351-23-982-7703.

E-mail address: reva@qui.uc.pt (I.D. Reva).
}

responds to the equilibrium ratio of conformers characteristic of the gaseous phase prior to matrix deposition. However, systems with low barriers to intramolecular rotations are exceptions to this rule [1]. In such systems additional conformational cooling may happen in the matrix. Here, as suggested by Felder and Günthard [2], the term 'conformational cooling' is used to describe processes in which 'populations of conformational energy levels are shifted to states of lower energy, but without requiring the existence of a thermodynamic temperature'.

The possibility of conformational cooling is not always taken into account in low temperature matrix isolation experiments. The assumption that 
individual conformers are trapped into cryogenic matrixes without alteration of their relative populations at the temperature conditions existing immediately before deposition of the gaseous sample [3] is often assumed a priori. For example, to probe terminal conformer populations in supersonic beams, Felder and Günthard [2] used matrix IR spectroscopy and the 'conformer-trapping assumption'. From their experiments they concluded that molecules with low (less than ca. $8 \mathrm{~kJ} \mathrm{~mol}^{-1}$ ) energy barriers to internal rotation might undergo conformational cooling by supersonic expansion. However, they did not consider the possibility of conformational cooling either at the arrival of the molecules on the cold substrate during matrix deposition or in the matrix itself.

An example of conformational cooling in matrixes was observed for glycine. In early matrix isolation studies of glycine [4] the samples were deposited at $20 \mathrm{~K}$ and only two conformers of glycine were observed. However, later on we showed that the three lowest energy conformers of glycine could be successfully trapped in argon matrixes when the substrate temperature was $13 \mathrm{~K}$ or lower [5,6]. At higher substrate temperatures the conversion of conformer III into the lowest energy conformer I occurs during the matrix deposition and this is why only two forms were observed at $20 \mathrm{~K}$.

Another compound undergoing conformational cooling in matrixes is methyl cyanoacetate (MCA), which we have recently studied [7]. According to the $\mathrm{MP} 2 / 6-31 \mathrm{G}^{* *}$ calculations, in the gaseous phase this molecule has two almost isoenergetic conformers differing in the relative orientation of the cyano and methylcarboxyl groups. We noticed that the relative populations of the two conformational states trapped in the matrixes strongly depended on the temperature of the optical substrate during deposition, unequivocally indicating that conformational cooling was taking place.

The results obtained in our study on MCA impelled us to consider other molecular systems where conformational cooling effects could also occur. Among the possible systems, cyanoacetic acid (CAA) appeared as a logical candidate due to its structural similarity to MCA. Indeed, the Hartree-Fock 6-31G* calculations [8] predicted that the structures of the two lowest energy minima of CAA are similar to those of the most stable conformers of MCA.

The aim of this work is to provide a further contribution to the explanation of the conformational cooling effects in low temperature matrixes as exemplified by the experimental data obtained for the first time for CAA. In the study we also supplied new data complementary to the available information [7] for the closely related molecule, MCA.

\section{Experiment}

The low temperature equipment, based on an APD Cryogenics closed-cycle helium refrigerator with DE-202A expander, is able to attain the lowest temperature of ca. $8 \mathrm{~K}$. This predefines the set of rare gases ( $\mathrm{Xe}, \mathrm{Ar}$, and $\mathrm{Kr}$ ) suitable for matrix experiments in this setup. Argon N60, krypton N48 and xenon N45 were supplied by Air Liquide. Commercial CAA and MCA were deposited onto a cold CsI window from a Knudsen cell through a needle valve kept at room temperature. Temperature was measured directly at the sample holder by a silicon diode temperature sensor connected to a Digital Temperature Controller (Scientific Instruments, Model 9650-1), which provides accuracy of $0.1 \mathrm{~K}$. Other details of the experimental setup can be found elsewhere [9].

\section{Theoretical calculations}

The equilibrium geometries of the low energy conformers of CAA and MCA were fully optimized at the DFT and MP2 levels of theory with the standard aug-cc-pVDZ basis set. Both methods were also used to obtain the reaction pathways for conformational interconversion and to locate the transition states. The DFT calculations were carried out with the three-parameter density functional abbreviated as B3LYP which includes Becke's gradient exchange correction [10], Lee et al. [11] and Vosko et al. [12] correlation functionals. All calculations were done on SGI ORIGIN 2000 and IBM Power 3 workstations using the Gaussian 98 program [13]. 


\section{Results and discussion}

CAA and MCA differ only in the substituent at one of the oxygen atoms (proton or methyl group). The most flexible internal degree of freedom in both compounds corresponds to the dihedral $\mathrm{C}-\mathrm{C}-\mathrm{C}=\mathrm{O}$ angle that describes the internal rotation around the central $\mathrm{C}-\mathrm{C}$ bond. A preliminary estimation of the barrier to the gauche $\rightarrow$ cis conversion (rotation around $\mathrm{C}-\mathrm{C}-\mathrm{C}=\mathrm{O}$ dihedral angle) in CAA gave the value of $3.4 \mathrm{~kJ}$ $\mathrm{mol}^{-1}$ (MP2/6-31++G** results). This value is essentially the same as in MCA $\left(3.6 \mathrm{~kJ} \mathrm{~mol}^{-1}\right)$ [7]. As discussed in Section 1, a barrier of $3 \mathrm{~kJ} \mathrm{~mol}^{-1}$ is low enough to allow conformational interconversion in matrixes.

The mechanism of the expected conformational conversion in both molecules is very simple, since the compounds have only two populated conformational states in the gaseous phase (gauche and cis). It involves simple one-step transformation of one conformer into another. For MCA we found that deposition of a sample at the temperature of the optical substrate equal to $20 \mathrm{~K}$ results in trapping of both conformers in the matrix (Fig. 1a, upper trace) and the gauche form dominates. However upon increasing of the substrate temperature the conformational cooling strongly shifts the MCA population towards the cis form (Fig. 1a, lower trace).

The lowest energy states of CAA and MCA have similar structures corresponding to the cis and gauche conformers (see Fig. 1). In both systems these states were predicted to have relative energies within $1 \mathrm{~kJ} \mathrm{~mol}^{-1}$. Thus we had been expecting the relative amount of the two conformers trapped in matrixes prepared using identical conditions to be very similar for the two compounds. However, we have noticed a striking difference between the two systems in our very first experiment with CAA. In that experiment, CAA was deposited into a xenon matrix at $20 \mathrm{~K}$. Thus both the matrix gas and the substrate temperature were exactly the same as in the case of MCA. To our surprise, in that experiment we observed bands due to only one conformer (Fig. 1b, lower trace). This result contradicted our expectations since, according to the calculated relative energies, the $c i s$
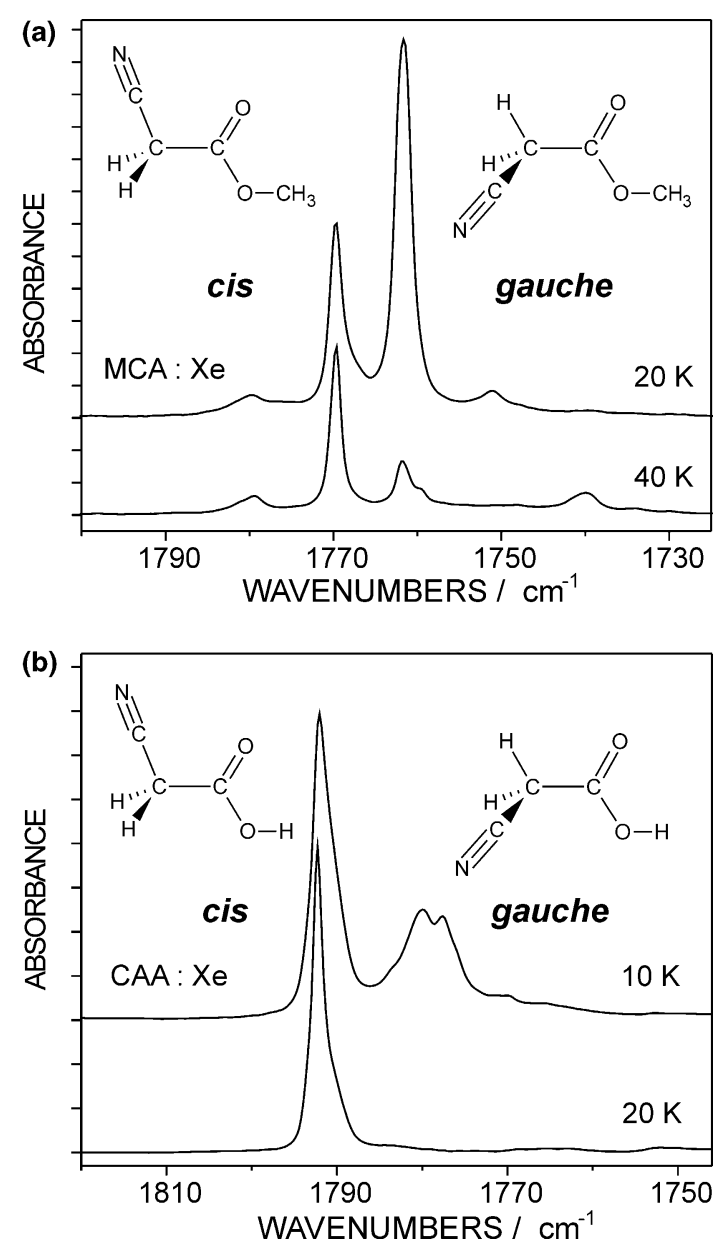

Fig. 1. The $\mathrm{C}=\mathrm{O}$ stretching region in the experimental FTIR spectra of MCA (a) and CAA (b) isolated in xenon matrixes. The temperature of the optical substrate during deposition of the samples was kept at 20 and $40 \mathrm{~K}$ for MCA and 10 and $20 \mathrm{~K}$ for CAA. The spectra normalized to the same intensity of the cis form.

and gauche conformers of CAA should coexist in the gaseous phase in comparable amounts. In order to elucidate the observed phenomenon we varied several parameters in the subsequent experiments. By decreasing the temperature of the optical substrate during matrix deposition we slowed the process of the conformational cooling and were able to freeze the second conformer into matrixes (Fig. 1b, upper trace).

In the additional experiments, several matrixes of CAA in argon, krypton and xenon were pre- 
pared using different substrate temperatures and their spectra were registered. Two sets of IR-bands related to the two gas phase conformers could be easily differentiated in the experiment (Fig. 2). Based on the comparison of the relative positions of absorptions in the experimental and calculated spectra, these two sets of bands were attributed to the gauche and the cis forms. For all the matrix gases, an increase of the substrate temperature resulted in a decrease of the relative population of the trapped gauche conformer with respect to the cis conformer. Thus, it is unequivocal that the cis form is the ground state form in the matrixes (it persists at all conditions), and the gauche form is a higher energy conformer of CAA.

We estimated the relative abundances ([gauche]/ $[c i s])$ of the two conformers in matrixes. The ratio of the experimental integral band intensities $\left(I_{\text {gauche }} / I_{\text {cis }}\right)$ was reduced using the calculated absolute intensities $\left(A_{\text {cis }}^{\text {th }} / A_{\text {gauche }}^{\text {th }}\right)$ for the corresponding vibration, so that:

$[$ gauche $] /[$ cis $]=\left(A_{\text {cis }}^{\text {th }} / A_{\text {gauche }}^{\text {th }}\right) \cdot\left(I_{\text {gauche }} / I_{\text {cis }}\right)$.

The result of the estimation made on the basis of the carbonyl stretching bands is presented in Fig. 3.

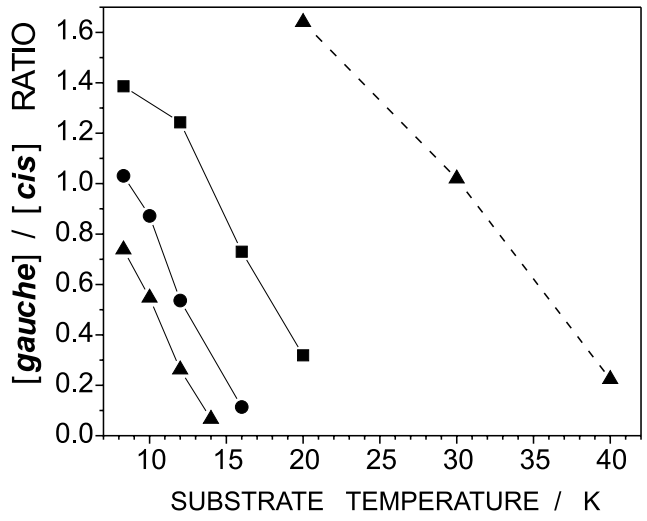

Fig. 3. Ratio between the gauche and cis conformers of CAA (solid lines) and MCA (dashed line) trapped in inert matrixes at different substrate temperatures. Estimation from the experimental integral band intensities in the carbonyl stretching region. The experimental intensities were reduced to the absolute intensities of the carbonyl stretching predicted at the MP2/augcc-pVDZ level (see Eq. (1)). Each point in the plot corresponds to a newly deposited matrix. Matrix gases: squares - argon; circles - krypton; triangles - xenon.

The estimation over other spectral regions produced similar results. At similar deposition temperatures, the relative amount of the minor

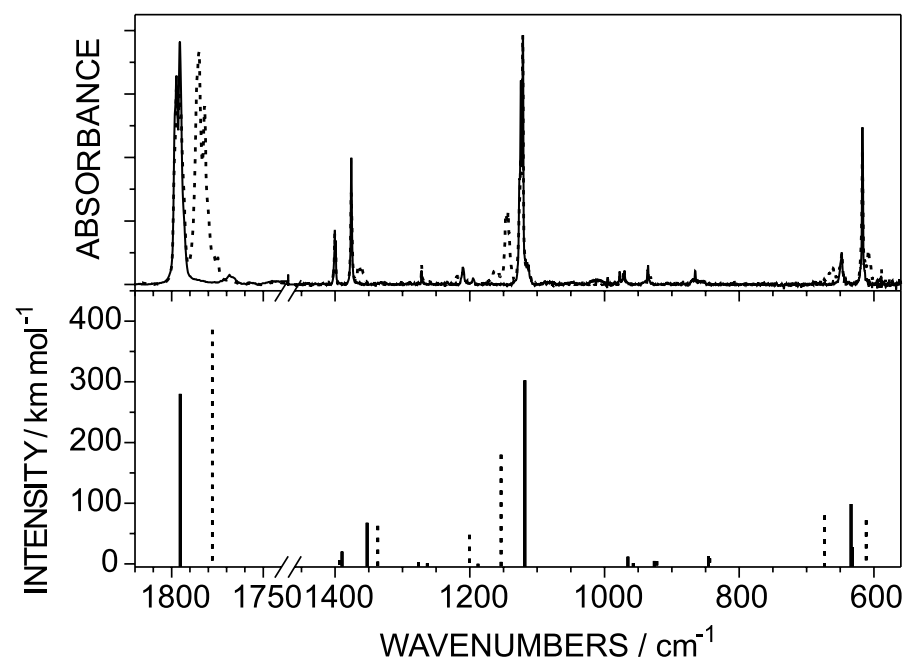

Fig. 2. Fingerprint region in the spectrum of CAA. Top - CAA in krypton matrix deposited from vapor kept at room temperature with the optical substrate kept at: solid line, $20 \mathrm{~K}$; dashed line, $8 \mathrm{~K}$. Spectra normalized to the same intensity of the strongest bands (e.g., 1797, 1400,1121, $648 \mathrm{~cm}^{-1}$ ) of the most stable conformer. The gauche conformer completely transforms in the matrix into the cis form, if the temperature of the optical substrate is increased. Bottom - spectra calculated at the DFT(B3LYP)/aug-cc-pVDZ level of theory. Wave numbers in the calculated spectra are scaled with a uniform factor of 0.98 for both conformers. Solid bars, cis; dashed bars, gauche conformer. 
conformer increases from xenon to argon. Its maximal observed concentration was obtained in an argon matrix at ca. $8 \mathrm{~K}$ - the lowest attainable temperature of the optical substrate in our setup. Interestingly, this result is in a good qualitative agreement with the study of Ruoff et al. [14], who showed that the same trend appears in seeded supersonic jets of inert gases. They found that krypton is able to cool species with higher barriers better than argon. In addition, they also pointed out that weaker relaxants such as helium and neon can be employed to efficiently trap higher energy conformers. The higher dipole moments of the cis form (5.28 D versus 2.57 D for the gauche form in CAA) may also contribute to a more efficient trapping of this form in the more polarizable matrixes.

The abundance of the gauche conformer at the lowest temperature in an argon matrix for CAA and in a xenon matrix for MCA significantly exceeds the abundance of the cis form (Fig. 3). For two forms approximately equal in energy this can only occur if one of them is degenerated by symmetry and another is not. This indeed occurs for the two compounds studied here as can be inferred from their calculated potential energy profile in the gas phase (Fig. 4). In both compounds the planar cis conformer co-exists with two symmetrically identical gauche conformers. The calculations also show that the barriers for intramolecular rotation from the gauche to the cis form (the direction of conformational cooling observed in the experiment for both compounds) are almost identical for CAA and MCA and equal to ca. $3 \mathrm{~kJ} \mathrm{~mol}^{-1}$ (Fig. 4).

Along with the similarities mentioned, the theoretical calculations (MP2/aug-cc-pVDZ level) revealed an important difference between the two compounds. The ground state form in CAA is the planar cis structure, while in MCA it is the gauche form. The energy gap between the two forms in each case is the same and equal to $0.86 \mathrm{~kJ} \mathrm{~mol}^{-1}$ (with account for the zero-point vibrational energies) [15]. This order of energies implies that in the equilibrium gaseous phase at room temperature the gauchelcis conformational ratio in CAA should be equal to $58.5 / 41.5=1.41$ (it is more than unity because the higher energy gauche form is

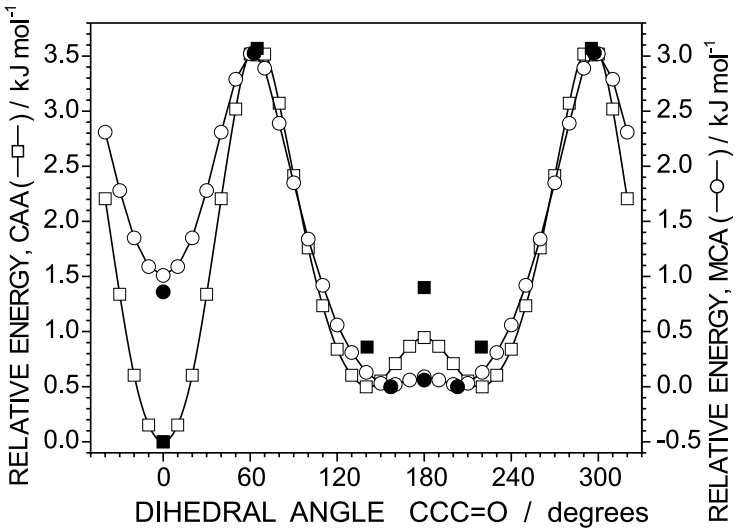

Fig. 4. Potential energy profile for the internal $\mathrm{C}-\mathrm{C}-\mathrm{C}=\mathrm{O}$ rotation in CAA and MCA calculated at the MP2/aug-ccpVDZ level of theory. For each point the chosen value for the $\mathrm{C}-\mathrm{C}-\mathrm{C}=\mathrm{O}$ torsional angle was kept constant while all other structural parameters were optimized. Open circles and squares, relaxed PES scan; filled circles and squares, relative position of the stationary points with account for the zero-point vibrational energy (with one lowest frequency excluded). Note that CAA and MCA have different ordinates; the scale along these axes is identical but zero-position is shifted to level out energies of the gauche forms. Such representation demonstrates clearly that the height of the barrier for the internal rotation from the gauche to the cis form is equal in both compounds.

doubly degenerated by symmetry), while in MCA it should be $73.9 / 26.1=2.83$. In the case of CAA the theoretically predicted ratio perfectly matches the experimentally found ratio of the conformers frozen in argon matrix at $9 \mathrm{~K}$ (Fig. 4, upper left square), and probably is close to the ratio of the conformational equilibrium inherent to the gaseous phase at the room temperature. In MCA, however, the ratio of the conformers frozen in xenon at $20 \mathrm{~K}$ hardly approaches 1.7 , indicating that partial conversion of the gauche into the cis form takes place during the matrix deposition.

It is interesting to estimate the gauche-to-cis ratio in MCA at the lowest temperature of the optical substrate attainable in our experimental setup $(8 \mathrm{~K})$, because this experimental condition gives the closest match to the conformer ratio in the gaseous phase. The linear extrapolation of the dashed line in Fig. 3 (xenon matrix, MCA) to $8 \mathrm{~K}$ gives the value close to 2.5 - still lower than the theoretically expected 2.83 . However, as the results for CAA demonstrate, the depopulation of the 
gauche form happens to a lesser extent in an argon matrix than in a xenon one. Thus, to approach the gaseous phase characteristic distribution, MCA was isolated at $8 \mathrm{~K}$ in an argon matrix.

The bands due to the absorptions of the cis and gauche forms of MCA in an argon matrix are broader than in xenon and they overlap. However a rough estimation of the relative cis/gauche population can still be made. Let us assume that the integral band intensities are proportional to the peak intensities (the full band widths at half maximum are approximately the same). In the freshly prepared matrix they are equal to 0.29 and 1.24 for the cis and gauche form, respectively (Fig. 5). The theoretical carbonyl intensities of these forms for MCA calculated at the MP2/augcc-pVDZ level are equal to 179.8 and $257.8 \mathrm{~km}$ $\mathrm{mol}^{-1}$, respectively. Thus using Eq. (1), the gauchel cis ratio for MCA trapped in an argon matrix at 8 $\mathrm{K}$ is estimated to be 2.98 . This ratio is close to the equilibrium ratio in the gaseous phase. This rough estimation gives the energy gap of about $1 \mathrm{~kJ}$ $\mathrm{mol}^{-1}$ in favor of the gauche form, which is in a very good agreement with the theoretically predicted difference in energy of $0.86 \mathrm{~kJ} \mathrm{~mol}^{-1}$ in MCA. The annealing (dashed line, Fig. 5) results in the growth of the peak at $1777.5 \mathrm{~cm}^{-1}$ (cis) and decrease of the doublet at 1770.5 and $1768.5 \mathrm{~cm}^{-1}$ (gauche). The observed direction of annealing

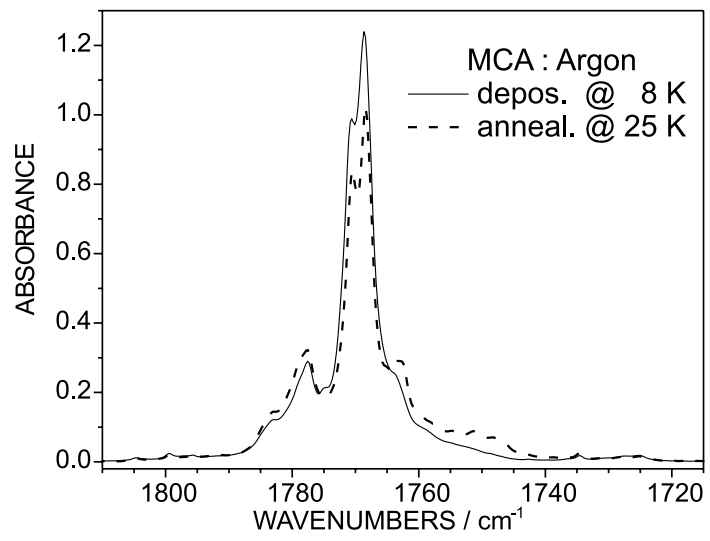

Fig. 5. The carbonyl stretching region in the FTIR spectrum of MCA isolated in an argon matrix at $8 \mathrm{~K}$ (solid line) and annealed at $25 \mathrm{~K}$ (dashed line). The sample was deposited from the vapor kept at room temperature. confirms that the cis form becomes the ground energy form in the matrix medium.

The two previous experimental IR studies of MCA revealed different conformers as the ground state energy forms. In the gaseous phase study [16], on the base of asymmetric top contour simulations, the preference was given to the gauche form. In the matrix isolation study [7], on the base of the annealing results, the choice fell upon the cis form. The theoretical calculations carried out at MP2/ $631 \mathrm{G}^{* *}$ level [7], could not resolve the contradiction, because the two calculated minima were close in energy. The newly presented theoretical results (Fig. 4) speak in favor of the gauche form as the ground state in MCA, and confirm the conclusions of the gaseous phase study [16]. However, the conclusions of the matrix study also remain valid! It is unequivocally the cis form that persists in matrixes after annealing. This phenomenon can be explained by the interaction of the two forms with the matrix. The cis form of MCA is stabilized by the matrix environment more than the gauche form leading to inversion of the relative stability of the conformers. One possible reason of the higher stabilization of the cis form in the matrixes could be the higher molecular dipole moment of the cis form $(5.86 \mathrm{D})$, than of the gauche form $(2.68 \mathrm{D})$.

In the case of CAA the order of stability of the two conformers remains the same in the theoretical calculations and in the experiment. Similarly to MCA, where the cis conformer is additionally stabilized in matrixes, the stabilization of the cis form should take place in CAA. This is evidenced by the strong shift of conformational populations in matrixes in favor of the cis form.

MCA and CAA have different theoretically predicted relative gauche/cis ratios in the gaseous phase (2.83 in MCA versus 1.41 in CAA) and the opposite order of stabilities of the two conformers. Thus there are different starting points to the subsequent shifts of conformational populations in matrixes during conformational cooling. This in part explains the initial experimental observation, which stimulated this study: that at $20 \mathrm{~K}$ in a xenon matrix the gauche $\rightarrow$ cis conversion for CAA is complete but for MCA it is not. It is also important to point out that the comparison of the results for CAA with those previously obtained for MCA 
[7] clearly demonstrates that molecules with closely related conformational characteristics in the gas phase (structurally identical conformers and energy barriers for conformational isomerization) may undergo considerably different conformational cooling in matrix isolation experiments. Hence, we can conclude that conformational cooling in a matrix experiment seems to be system dependent, i.e., sensitive to specific interactions between the host and guest molecules, and it does not appear to follow any general quantitative rule.

With a proper care in preparing the samples and in the analysis of the results, the matrix deposition appears to be an efficient method for conformational cooling of isomers separated by a relatively low energy barriers and can be used as an alternative to cooling in the supersonic free jet expansion [17-19]. The gaseous phase conformational equilibria are especially difficult to shift for the systems with close in energy conformers. The effect reported here may be used for suppressing the populations of certain components in such systems.

\section{Conclusions}

The results obtained in this study demonstrate that for molecules with non-equal conformational states separated by low energy barriers (a few kJ $\mathrm{mol}^{-1}$ ), the low temperature inert matrix distribution of the conformers may strongly differ from that existing in the equilibrium gaseous phase due to the effect of conformational cooling. The degree of the conformational cooling depends on the following factors: (i) nature of the matrix gas host. In the series xenon-krypton-argon, xenon has the strongest and argon has the weakest relaxant properties; (ii) nature of the matrix-isolated guest. For the two related compounds, CAA and MCA, with the same height of barrier for intramolecular rotation in the gaseous phase, conformational cooling at the same matrix conditions is substantially different because of (iii) relative energies and (iv) dipole moments of the conformers participating in the conformational cooling; (v) temperature of the optical substrate during the matrix deposition. The lower this temperature is, the closer the ratio of the conformers frozen in matrix resembles the equilibrium ratio of conformers existent in the gaseous phase prior to the matrix deposition.

\section{Acknowledgements}

The financial support of the Fundação para a Ciência e a Tecnologia, Lisbon (Grant FCT \# SFRH/BPD/1661/2000 and research project POCTI/43366/QUI/2001) is acknowledged. The contribution of SGS and LA to this project was supported by a NATO Grant PST.CLG.978377.

\section{References}

[1] A.J. Barnes, J. Mol. Struct. 113 (1984) 161.

[2] P. Felder, Hs.H. Günthard, Chem. Phys. 71 (1982) 9.

[3] M. Tasumi, M. Nakata, J. Mol. Struct. 126 (1985) 111.

[4] Y. Grenie, C. Garrigou-Lagrange, J. Mol. Spectrosc. 41 (1972) 240.

[5] I.D. Reva, A.M. Plokhotnichenko, S.G. Stepanian, A.Yu. Ivanov, E.D. Radchenko, G.G. Sheina, Yu.P. Blagoi, Chem. Phys. Lett. 232 (1995) 141, Ibid. 235, 617 (Erratum).

[6] S.G. Stepanian, I.D. Reva, E.D. Radchenko, M.T.S. Rosado, M. Duarte, R. Fausto, L. Adamowicz, J. Phys. Chem. A 102 (1998) 1041.

[7] I.D. Reva, S.V. Ilieva, R. Fausto, Phys. Chem. Chem. Phys. 3 (2001) 4235.

[8] I.G. Binev, B.A. Stamboliyska, Y.I. Binev, J. Mol. Struct. 444 (1998) 235.

[9] I.D. Reva, S.G. Stepanian, L. Adamowicz, R. Fausto, J. Phys. Chem. A 105 (2001) 4773.

[10] A.D. Becke, Phys. Rev. A 38 (1988) 3098.

[11] C.T. Lee, W.T. Yang, R.G. Parr, Phys. Rev. B 37 (1988) 785.

[12] S.H. Vosko, L. Wilk, M. Nusair, Can. J. Phys. 58 (1980) 1200.

[13] M.J. Frisch et al., Gaussian 98, Revision A.11.1, Pittsburgh PA, 2001.

[14] R.S. Ruoff, T.D. Klots, T. Emilsson, H.S. Gutowsky, J. Chem. Phys. 93 (1990) 3142.

[15] The accuracy in calculations of the relative energies of isomers plays an important role in the interpretation of our results. The energy effects resulting from the rotation around the $\mathrm{C}-\mathrm{C}$ bond in MCA and CAA should primarily involve changes in the electrostatic and dispersion interactions between the functional groups linked to the two rotating carbon atoms. Since the MP2 level of theory includes the electron correlation effects and diffuse orbitals are included in the basis, the majority of the dispersion interaction energy should be fairly well described in the calculations. Also, the MP2 method usually provides good 
description of the electron density distribution and, thus, it should fairly well describe the electrostatic interactions since they primarily involve interactions of the local dipoles of the functional groups attached to the rotating carbons. In conclusion, although it is perhaps unrealistic to assume that the $1 \mathrm{~kJ} \mathrm{~mol}^{-1}$ accuracy can be achieved with the MP2/ aug-cc-PVDZ method, the results should not be far from this target.
[16] S.J. Leibowitz, J. Laane, C. Van Alsenoy, B.J. van der Veken, J. Mol. Struct. 248 (1991) 251.

[17] P.D. Godfrey, R.D. Brown, J. Am. Chem. Soc. 120 (1998) 10724.

[18] P.D. Godfrey, R.D. Brown, F.M. Rodgers, J. Mol. Struct. 376 (1996) 65.

[19] P.D. Godfrey, F.M. Rodgers, R.D. Brown, J. Am. Chem. Soc. 119 (1997) 2232. 